\title{
Univalent and Starlike Properties for Generalized Struve Function
}

\author{
Aaisha Farzana Habibullah, ${ }^{1}$ Adolf Stephen Bhaskaran, ${ }^{1}$ \\ and Jeyaraman Muthusamy Palani ${ }^{2}$
}

${ }^{1}$ Department of Mathematics, Madras Christian College, Tambaram, Chennai, Tamil Nadu 600 059, India

${ }^{2}$ Department of Mathematics, L. N. Government College, Ponneri, Chennai, Tamil Nadu 601 204, India

Correspondence should be addressed to Aaisha Farzana Habibullah; h.aaisha@gmail.com

Received 10 April 2016; Accepted 22 May 2016

Academic Editor: Teodor Bulboaca

Copyright (C) 2016 Aaisha Farzana Habibullah et al. This is an open access article distributed under the Creative Commons Attribution License, which permits unrestricted use, distribution, and reproduction in any medium, provided the original work is properly cited.

We derive conditions on the parameters $p, b$, and $c$ so that the function $z w_{p, b, c}(z)$, where $w_{p, b, c}(z)$ is the normalized form of generalized Struve function, belongs to the class $S_{1}^{*}(\alpha)$. Also, some sufficient conditions for the function $z w_{p, b, c}(z)$, to be in the class $\mathcal{U}(\lambda)$, are obtained.

\section{Introduction and Preliminaries}

Let $\mathbb{U}:=\{z:|z|<1\}$ denote the unit disc in the complex plane $\mathbb{C}$ and let $\mathscr{A}$ denote the class of functions which are analytic and of the form

$$
f(z)=z+\sum_{n=2}^{\infty} a_{n} z^{n}
$$

and normalized by the conditions $f(0)=0$ and $f^{\prime}(0)=1$. Let $\mathcal{S}$ denote the class of functions such that

$$
\mathcal{S}=\{f: f \in \mathscr{A} \text { and } f \text { univalent in } \mathbb{U}\} .
$$

Suppose that $f$ and $g$ are two analytic functions in $\mathbb{U}$, and $g$ is univalent in $\mathbb{U}$. We say that $f$ is subordinate to $g$, written $f(z) \prec g(z)$ or $f<g$, if and only if $f(0)=g(0)$ and $f(\mathbb{U}) \subset$ $g(\mathbb{U})$.

A function $f \in \mathscr{A}$ belongs to the class of starlike functions of order $\alpha$ denoted by $S^{*}(\alpha)$ if

$$
\operatorname{Re}\left(\frac{z f^{\prime}(z)}{f(z)}\right)>\alpha, \quad z \in \mathbb{U}
$$

A subclass of the class of starlike functions denoted by $S_{1}^{*}(\alpha)$ for $0 \leq \alpha<1$ consists of functions for which

$$
\left|\frac{z f^{\prime}(z)}{f(z)}-1\right|<1-\alpha, \quad z \in \mathbb{U} \text {. }
$$

We also note that $S_{1}^{*}(0) \subset S^{*}$. In [1-3] the authors have discussed the coefficient bounds and other extremal properties of the class $S_{1}^{*}(\alpha)$.

For $0<\lambda \leq 1$, consider the class

$\mathscr{U}(\lambda)$

$$
=\left\{f \in \mathscr{A}:\left|\left(\frac{z}{f(z)}\right)^{2} f^{\prime}(z)-1\right|<\lambda \text { for } z \in \mathbb{U}\right\} .
$$

From [4], we have the strict inclusion $\mathscr{U}(1):=\mathscr{U} \subset \mathcal{S}$. Very recently, Obradović et al. [5] have discussed the geometric behaviour of functions in $\mathscr{U}(\lambda)$. Also the class has been widely studied by many authors in $[4,6-8]$.

Let us consider the following second-order linear nonhomogenous differential equation (for more details see $[9,10]$ ):

$$
\begin{aligned}
& z^{2} u^{\prime \prime}(z)+b z u^{\prime}(z)+\left[c z^{2}-p^{2}+(1-b) p\right] u(z) \\
& \quad=\frac{4(z / 2)^{p+1}}{\sqrt{\pi} \Gamma(p+b / 2)},
\end{aligned}
$$


where $b, p, c \in \mathbb{C}$. The function $u_{p, b, c}(z)$ which is called the generalized Struve function of order $p$ is defined as a particular solution of (6) and has the series representation as follows:

$$
\begin{array}{r}
u_{p, b, c}(z) \\
=\sum_{n \geq 0} \frac{(-1)^{n}(c)^{n}}{\Gamma(n+3 / 2) \Gamma(p+n+(b+2) / 2)}\left(\frac{z}{2}\right)^{2 n+p+1}, \\
z \in \mathbb{C},
\end{array}
$$

where $\Gamma$ stands for the Euler gamma function.

Now, we consider the function $w_{p, b, c}(z)$ defined in terms of the generalized Struve function $u_{p, b, c}(z)$ by the transformation:

$$
w_{p, b, c}(z)=2^{P} \sqrt{\pi} \Gamma\left(p+\frac{b+2}{2}\right) z^{(-p-1) / 2} u_{p, b, c}(\sqrt{z}) .
$$

By using the well-known Pochammer symbol (or the shifted factorial) $(\chi)_{n}$ defined for $\chi, n \in \mathbb{C}$ in terms of the Euler $\Gamma$ function, we have

$$
\begin{aligned}
& (\chi)_{n}=\frac{\Gamma(\chi+n)}{\Gamma(\chi)} \\
& \quad= \begin{cases}1, & \text { if } n=0, \chi \in \mathbb{C}^{*}, \\
\chi(\chi+1) \cdots(\chi+n-1), & \text { if } n \in \mathbb{N}, \chi \in \mathbb{C} .\end{cases}
\end{aligned}
$$

We obtain the following series representation for the function $w_{p, b, c}$ given by (8):

$$
w_{p, b, c}(z)=\sum_{n \geq 0} \frac{(-c / 4)^{n}}{(3 / 2)_{n}(k)_{n}} z^{n}
$$

where $k=p+(b+2) / 2 \neq 0,-1,-2, \ldots$. Also, this function is analytic on $\mathbb{C}$ and satisfies the following second-order inhomogeneous differential equation:

$$
\begin{aligned}
& 4 z^{2} w^{\prime \prime}(z)+2(2 p+b+3) z w^{\prime}(z) \\
& +(c z+2 p+b) w(z)=(2 p+b),
\end{aligned}
$$

where $p, b, c \in \mathbb{C}$.

Recently, the class $\mathscr{U}(\lambda)$ and its generalizations have been widely studied by many authors $[4-8,11]$. By applying the admissible function method authors in [12] have obtained conditions on the triplet $a, b$, and $c$ such that $z F_{a, b, c}(z)$ is in the class $\mathcal{U}$, where $F_{a, b, c}(z)$ is the confluent hypergeometric function. In [13], the authors have derived conditions on the parameters $a, b$, and $c$ such that the function $z_{2} F_{1}(a, b, c)$ is in $S_{1}^{*}(0)$, where ${ }_{2} F_{1}(a, b, c ; z)$ is the Gaussian hypergeometric function. Moreover, in [9, 10] Yagmur and Orhan have obtained sufficient conditions for the generalized Struve function to be convex, starlike, and univalent. Most of these results were motivated by the research on geometric properties of Gaussian and confluent hypergeometric function.

Motivated by the above-mentioned works, in this paper we use the method of differential subordination to show that $z w_{p, b, c}(z)$ is in the class $S^{*}(\alpha)$ and also we provide sufficient conditions for the function $z w_{p, b, c}(z)$ to be in the class $\mathscr{U}$ and hence univalent.

To prove our main results, we will need the following lemmas.

Lemma 1 (see [14]). Let (nonconstant) function $p(z)$ be analytic in $\mathbb{U}$ with $p(0)=0, p(z) \neq 0(z \in \mathbb{U})$.

If $|p(z)|$ attains its maximum value on the circle $|z|=r<1$ at a point $z_{0} \in \mathbb{U}$, then

$$
\begin{aligned}
z_{0} p^{\prime}\left(z_{0}\right) & =k p\left(z_{0}\right), \\
\Re\left(1+\frac{z_{0} p^{\prime \prime}\left(z_{0}\right)}{p^{\prime}\left(z_{0}\right)}\right) & \geq k,
\end{aligned}
$$

where $k$ is a real number and $k \geq 1$.

Lemma 2 (see [15]). If an analytic function $f$ has the form $f(z)=z+a_{2} z^{2}+\cdots, z \in \mathbb{U}$ and satisfies the condition

$$
\left|\frac{z^{2} f^{\prime}(z)}{f^{2}(z)}-1\right|<1
$$

then $f$ is univalent in $\mathbb{U}$.

Lemma 3 (see [15]). Let $\beta$ be a complex number, $\mathfrak{R}(\beta)>0$, and let $\alpha$ be a complex number, $|\alpha| \leq 1, \alpha \neq-1$, and $h(z)=$ $z+a_{2} z^{2}+\cdots$ a regular function on $\mathbb{U}$. If

$$
\left.|\alpha| z\right|^{2 \beta}+\left(1-|z|^{2 \beta}\right) \frac{z h^{\prime \prime}(z)}{\beta h^{\prime}(z)} \mid \leq 1
$$

for all $z \in \mathbb{U}$, then the function

$$
\begin{aligned}
F_{\beta}(z)= & \left(\beta \int_{0}^{z} t^{\beta-1} h^{\prime}(t) d t\right)^{1 / \beta} \\
= & z+\frac{2 a_{2}}{(1+\beta)} z^{2} \\
& +\left(\frac{3 a_{3}}{2+\beta}+\frac{2 \beta(1-\beta) a_{2}^{2}}{(\beta+1)^{3}}\right) z^{3}+\cdots
\end{aligned}
$$

is regular and univalent in $\mathbb{U}$.

Lemma 4 (see [16]). Let $\Omega \subset \mathbb{C}$ and let $q$ be analytic and univalent on $\mathbb{U}$ except for those $\xi \in \partial \mathbb{U}$ for which $\lim _{z \in \xi} q(z)=$ $\infty$. Suppose that $\psi: \mathbb{C}^{3} \times \mathbb{U} \rightarrow \mathbb{C}$ satisfies the condition

$$
\psi\left(q(\zeta), m \zeta q^{\prime}(\zeta), \zeta^{2} q^{\prime \prime}(\zeta) ; z\right) \notin \Omega,
$$

where $q(z)$ is finite, $m \geq n \geq 1$, and $|\xi|=1$. If $p$ and $q$ analytic in $\mathbb{U}, p(z)=p(0)+p_{n} z^{n}+\cdots, p(0)=q(0)$, and further if

$$
\psi\left(p(z), z p^{\prime}(z), z^{2} p^{\prime \prime}(z) ; z\right) \in \Omega
$$

then $p(z) \prec q(z)$ in $\mathbb{U}$. 
Suppose that $p(z)$ is analytic in $\mathbb{U}$ with $p(z)=p_{n} z^{n}+$ $p_{n+1} z^{n+1}+\cdots$ and $q(z)=M z$. Then the condition (16) reduces to a simple form:

$$
\psi\left(M e^{i \theta}, K e^{i \theta}, L ; z\right) \notin \Omega
$$

whenever $K \geq n M, \operatorname{Re}\left(L e^{-i \theta}\right) \geq(n-1) K, z \in \mathbb{U}$, and $\theta \in \mathbb{R}$.

\section{Main Results}

Theorem 5. Let $\delta>0, p, b \in \mathbb{R}$, and $c \in \mathbb{C}$ such that $|c| \delta<$ $3[(2 p+b)+2]-|c| \delta ;$ then

$$
\left|w_{p, b, c}(\delta z)-1\right|<\frac{|c| \delta}{3[(2 p+b)+2]-|c| \delta}, \quad z \in \mathbb{U} .
$$

Proof. Let $p(z)=w_{p, b, c}(\delta z)-1, \delta>0$; then $p(z)$ is analytic in $\mathbb{U}$ with $p(0)=0$. Since the function $w_{p, b, c}(z)$ satisfies the differential equation (11) and $w_{p, b, c}(\delta z) \stackrel{=}{=} p(z)+1, p(z)$ satisfies the following inhomogeneous differential equation:

$$
\begin{aligned}
& 4 z^{2} p^{\prime \prime}(z)+2(2 p+b+3) z p^{\prime}(z) \\
& \quad+(c \delta z+2 p+b)(p(z)+1)=(2 p+b) .
\end{aligned}
$$

Using Lemma 4 , we will show that $|p(z)|<M$, where $M=|c| \delta / 3[(2 p+b)+2]-|c| \delta$. For this, if we let

$$
\begin{aligned}
\psi(r, s, t ; z)= & 4 t+2(2 p+b+3) s \\
& +(c \delta z+2 p+b)(1+r)-(2 p+b)
\end{aligned}
$$

and $\Omega=\{0\}$, then it is sufficient to prove that $\psi\left(M e^{i \theta}\right.$, $\left.K e^{i \theta}, L ; z\right) \notin \Omega$ whenever $K \geq M, \operatorname{Re}\left(L e^{-i \theta}\right) \geq 0, z \in \mathbb{U}$, and $\theta$ is real, we have that

$$
\begin{aligned}
\mid \psi & \left(M e^{i \theta}, K e^{i \theta}, L ; z\right)|=| 4 L+2(2 p+b+3) K e^{i \theta} \\
& +(c \delta z+2 p+b)\left(1+M e^{i \theta}\right)-(2 p+b)|=| 4 L e^{-i \theta} \\
& +2(2 p+b+3) K+(c \delta z+2 p+b)\left(M+e^{-i \theta}\right) \\
& -(2 p+b) e^{-i \theta} \mid \geq 4 \operatorname{Re}\left(L e^{-i \theta}\right)+2(2 p+b+3) M \\
& +\operatorname{Re}((c \delta z+2 p+b) M) \\
& +\operatorname{Re}\left((c \delta z+2 p+b) e^{-i \theta}\right)-\operatorname{Re}\left((2 p+b) e^{-i \theta}\right) \\
& \geq[2(2 p+b+3)+(2 p+b)] M-\operatorname{Re}\left(c \delta z e^{-i \theta}\right) \\
& -\operatorname{Re}(c \delta z M) \geq 3[(2 p+b)+2] M-|c| \delta M-|c| \delta \\
& =0 .
\end{aligned}
$$

In the last stage of the inequalities we have used the definition of $M$ and shown that

$$
\psi\left(M e^{i \theta}, K e^{i \theta}, L ; z\right) \notin \Omega=\{0\}
$$

whenever $K \geq M, \theta$ is real, and $z \in \mathbb{U}$. Hence, $|p(z)|<M$.
Choosing $\delta=1$ in the above theorem we have the following Corollary.

Corollary 6. Let $p, b \in \mathbb{R}$, and $c \in \mathbb{C}$ be such that $3[(2 p+b)+$ $2]-2|c|>0$; then

$$
\left|w_{p, b, c}(z)-1\right|<\frac{|c|}{3[(2 p+b)+2]-|c|}, \quad z \in \mathbb{U} .
$$

The next results give sufficient conditions for the function $g_{p, b, c}(z)=z w_{p, b, c}(z)$ to be starlike and univalent in the open unit disc.

Theorem 7. If $p, b \in \mathbb{R}$, and $c \in \mathbb{C}$ such that $2|c|<3[(2 p+$ b) +2$]$ and

$$
\begin{aligned}
& \frac{(2 p+b)|c|}{[3[(2 p+b)+2]-2|c|]}+|c| \\
& \quad \leq(1-\alpha)[2(2 p+b+3)-4(1-\alpha)],
\end{aligned}
$$

then the function $g_{p, b, c}(z)$ belongs to the class $S_{1}^{*}(\alpha)$.

Proof. For $g_{p, b, c}(z)=z w_{p, b, c}(z)$ to be in the class $S_{1}^{*}(\alpha)$ we need to prove that

$$
\left|\frac{z g_{p, b, c}^{\prime}(z)}{g_{p, b, c}}-1\right|<(1-\alpha), \quad z \in \mathbb{U} .
$$

Upon setting

$$
\frac{\left[z w_{p, b, c}(z)\right]^{\prime}}{w_{p, b, c}(z)}-1=\widetilde{q}(z)
$$

we observe that $\widetilde{q}(z)$ is analytic in $\mathbb{U}$ and $\widetilde{q}(0)=\widetilde{q}^{\prime}(0)-1=0$. Also

$$
\begin{aligned}
z w_{p, b, c}^{\prime}(z) & =\widetilde{q}(z) w_{p, b, c}(z), \\
z^{2} w_{p, b, c}^{\prime \prime}(z) & =[(\widetilde{q}(z)-1) \tilde{q}(z)+z \widetilde{q}(z)] w_{p, b, c}(z) .
\end{aligned}
$$

Now, since $w_{p, b, c}(z)$ satisfies the inhomogeneous differential equation (11), in terms of $\widetilde{q}(z)$ we see that $\tilde{q}(z)$ satisfies the following equation:

$$
\psi\left(\widetilde{q}(z), z \widetilde{q}^{\prime}(z) ; z\right)=0,
$$

where

$$
\begin{aligned}
\psi(r, s ; z)= & 4[r(r-1)+s]+2(2 p+b+3) r \\
& +(c z+2 p+b)-(2 p+b) \frac{1}{g_{p, b, c}(z)} .
\end{aligned}
$$

Now, we claim that $|\widetilde{q}(z)|<1-\alpha, 0 \leq \alpha<1$. By using Lemma 4 with $\Omega=\{0\}, n=1$, and $q(z)=(1-\alpha) z$, we need to show that

$$
\psi\left((1-\alpha) e^{i \theta}, K e^{i \theta} ; z\right) \notin \Omega,
$$


where $\theta$ is real, $K \geq(1-\alpha)$, and $z \in \mathbb{U}$ :

$$
\psi\left((1-\alpha) e^{i \theta}, K e^{i \theta} ; z\right)=e^{i \theta} C-D,
$$

where

$$
\begin{aligned}
C= & 4\left[(1-\alpha)\left[(1-\alpha) e^{i \theta}-1\right]+K\right] \\
& +2(2 p+b+3)(1-\alpha), \\
D= & (2 p+b)\left[\frac{1}{g_{p, b, c}(z)}-1\right]-c z .
\end{aligned}
$$

Also, when $\delta=1$ in Theorem 5 we have that if $2|c|<3(2 p+$ b) +6 then

$$
\left|w_{p}(z)-1\right|<\frac{|c|}{3(2 p+b)+6}-|c| \leq 1 .
$$

Also,

$$
\begin{aligned}
|C| & \geq \operatorname{Re} C>4(1-\alpha)\left[(1-\alpha) e^{i \theta}-1\right]+(1-\alpha) 4 \\
& +2(2 p+b+3)(1-\alpha) \geq(1-\alpha)[4(1-\alpha) \cos \theta \\
& -4(1-\alpha)+4(1-\alpha)+2(2 p+b+3)] \geq(1-\alpha) \\
& \cdot[2(2 p+b+3)-4(1-\alpha)]=\widetilde{\beta}, \text { say. }
\end{aligned}
$$

Now, if we show that $|D|<\widetilde{\beta}$, then we have

$$
|\psi(r, s ; z)| \geq\left|e^{i \theta} C-D\right| \geq|C|-|D|=0 .
$$

In order to establish that we need the following results which state that for $r<1$ and $|w-1|<r$ if and only if

$$
\left|\frac{1}{w}-\frac{1}{1-r^{2}}\right|<\frac{r}{1-r^{2}}
$$

In particular, $|w-1|<r$ implies that

$$
\left|\frac{1}{w}-1\right|<\frac{r}{1-r}
$$

When $r=|c| /(3[(2 p+b)+2]-|c|)$, we have

$$
\left|\frac{1}{g_{p, b, c}(z)}-1\right|<\frac{r}{1-r}=\frac{|c|}{3(2 p+b)+6-2|c|} \text {. }
$$

Using the last inequality, we have

$$
|D| \leq(2 p+b)\left|\frac{1}{g_{p, b, c}(z)}-1\right|+|c||z|
$$

$$
<\frac{(2 p+b)|c|}{3[(2 p+b)+2]-2|c|}+|c|
$$

and so we have $|D|<\widetilde{\beta}$ provided that

$$
\frac{(2 p+b)|c|}{3[(2 p+b)+2]-2|c|}+|c| \leq \widetilde{\beta}
$$

holds, where $\tilde{\beta}$ is given by (35). And, we observe that the above condition is stated in the theorem. Thus, $|\widetilde{q}(z)|<(1-\alpha)$ in $\mathbb{U}$ and hence $g_{p, b, c}(z)$ belongs to $S_{1}^{*}(\alpha)$.

Theorem 8. Let $p, b \in \mathbb{R}$, and $c \in \mathbb{C}$. If $g_{p, b, c}(z)$ satisfies any one of the following inequalities:

$$
\begin{gathered}
\left|\frac{z^{2} g_{p, b, c}^{\prime}(z)}{\left[g_{p, b, c}(z)\right]^{2}}\left[\frac{\left(z g_{p, b, c}(z)\right)^{\prime \prime}}{g_{p, b, c}^{\prime}(z)}-\frac{2 z g_{p, b, c}^{\prime}(z)}{g_{p, b, c}(z)}\right]\right|<1 \quad(z \in \mathbb{U}), \\
\left|\frac{\left[g_{p, b, c}(z)\right]^{2}}{z^{2} g_{p, b, c}^{\prime}(z)}\left[\frac{\left[z g_{p, b, c}(z)\right]^{\prime \prime}}{g_{p, b, c}^{\prime}(z)}-\frac{2 z g_{p, b, c}^{\prime}(z)}{g_{p, b, c}(z)}\right]\right|<\frac{1}{4} \quad(z \in \mathbb{U}), \\
\operatorname{Re}\left[\frac{\left[z g_{p, b, c}(z)\right]^{\prime \prime} / g_{p, b, c}^{\prime}(z)-2 z g_{p, b, c}^{\prime}(z) / g_{p, b, c}(z)}{z^{2} g_{p, b, c}^{\prime}(z) /\left(g_{p, b, c}(z)\right)^{2}-1} \mid<\frac{1}{2} \quad(z \in \mathbb{U}),\right. \\
\left(g_{p, b, c}(z)\right)^{2}\left[\frac{\left[z g_{p, b, c}(z)\right]^{\prime \prime} / g_{p, b, c}^{\prime}(z)-2 z g_{p, b, c}^{\prime}(z) / g_{p, b, c}(z)}{z^{2} g_{p, b, c}^{\prime}(z) /\left[g_{p, b, c}(z)\right]^{2}}\right] \mid<1 \quad(z \in \mathbb{U}),
\end{gathered}
$$

then $g_{p, b, c}$ is in $\mathcal{U}$ and hence $g_{p, b, c}(z)$ is univalent in $\mathbb{U}$. 
Proof. Define a function $p(z)$ by

$$
\frac{z^{2} g_{p, b, c}^{\prime}(z)}{\left[g_{p, b, c}(z)\right]^{2}}=1+p(z) \quad(z \in \mathbb{U})
$$

and then $p(z)$ is analytic in $\mathbb{U}$ and $p(0)=0$. Differentiating (46) gives

$$
\frac{\left[z g_{p, b, c}(z)\right]^{\prime \prime}}{g_{p, b, c}^{\prime}(z)}-\frac{2 z g_{p, b, c}^{\prime}(z)}{g_{p, b, c}(z)}=\frac{z p^{\prime}(z)}{1+p(z)}
$$

Hence, from (46) and (47), we have

$$
\begin{aligned}
& E_{1}(z)=\frac{z^{2} g_{p, b, c}^{\prime}(z)}{\left(g_{p, b, c}(z)\right)^{2}}\left(\frac{\left[z g_{p, b, c}(z)\right]^{\prime \prime}}{g_{p, b, c}^{\prime}(z)}-\frac{2 z g_{p, b, c}^{\prime}(z)}{g_{p, b, c}(z)}\right)=z p^{\prime}(z), \\
& E_{2}(z)=\frac{\left(g_{p, b, c}(z)\right)^{2}}{z^{2} g_{p, b, c}^{\prime}(z)}\left(\frac{\left[z g_{p, b, c}(z)\right]^{\prime \prime}}{g_{p, b, c}^{\prime}(z)}-\frac{2 z g_{p, b, c}^{\prime}(z)}{g_{p, b, c}(z)}\right)=\frac{z p^{\prime}(z)}{(1+p(z))^{2}}, \\
& E_{3}(z)=\frac{\left[z g_{p, b, c}(z)\right]^{\prime \prime} / g_{p, b, c}^{\prime}(z)-2 z g_{p, b, c}^{\prime}(z) / g_{p, b, c}(z)}{z^{2} g_{p, b, c}^{\prime}(z) /\left(g_{p, b, c}(z)\right)^{2}-1}=\frac{z p^{\prime}(z)}{p(z)} \frac{1}{(1+p(z))}, \\
& E_{4}(z)=\frac{z^{2} g_{p, b, c}^{\prime}(z)}{\left(g_{p, b, c}(z)\right)^{2}}\left(\frac{\left[z g_{p, b, c}(z)\right]^{\prime \prime} / g_{p, b, c}^{\prime}(z)-2 z g_{p, b, c}^{\prime}(z) / g_{p, b, c}(z)}{z^{2} g_{p, b, c}^{\prime}(z) /\left(g_{p, b, c}(z)\right)^{2}-1}\right)=\frac{z p^{\prime}(z)}{p(z)} .
\end{aligned}
$$

Now suppose that there exists $z_{0} \in \mathbb{U}$ such that

$$
\max _{|z|<\left|z_{0}\right|}|p(z)|=\left|p\left(z_{0}\right)\right|=1,
$$

and then from Lemma 1, we have

$$
z_{0} p^{\prime}\left(z_{0}\right)=k p\left(z_{0}\right) \text {. }
$$

Therefore, letting $p\left(z_{0}\right)=e^{i \theta}$ in each of (48), we obtain that

$$
\begin{aligned}
\left|E_{1}\left(z_{0}\right)\right| & =\left|z_{0} p^{\prime}\left(z_{0}\right)\right|=\left|k e^{i \theta}\right| \geq 1, \\
\left|E_{2}\left(z_{0}\right)\right| & =\left|\frac{z_{0} p^{\prime}\left(z_{0}\right)}{\left(1+p\left(z_{0}\right)\right)^{2}}\right|=\frac{k}{\left|1+e^{i \theta}\right|^{2}} \geq \frac{1}{4}, \\
\left|E_{3}\left(z_{0}\right)\right| & =\left|\frac{z_{0} p^{\prime}\left(z_{0}\right)}{p\left(z_{0}\right)} \frac{1}{1+p\left(z_{0}\right)}\right|=\frac{k}{\left|1+e^{i \theta}\right|} \geq \frac{1}{2}, \\
\operatorname{Re}\left\{E_{4}\left(z_{0}\right)\right\} & =\operatorname{Re}\left\{\frac{z_{0} p^{\prime}\left(z_{0}\right)}{p\left(z_{0}\right)}\right\}=k \geq 1,
\end{aligned}
$$

which contradicts our assumption (42)-(45), respectively. Therefore, $|w(z)|<1$ for all $z \in \mathbb{U}$; then from (46) we have

$$
\left|\frac{z^{2} g_{p, b, c}^{\prime}(z)}{\left(g_{p, b, c}(z)\right)^{2}}-1\right|=|w(z)|<1
$$

which implies $g_{p, b, c}$ is in the class $\mathcal{U}$ and hence univalent.
Theorem 9. Let $c>0, d \geq 0$, such that $c+2 d \leq 1$. If $g_{p, b, c}(z)$ satisfies the inequality

$$
\Re\left\{\frac{\left[z g_{p, b, c}(z)\right]^{\prime \prime}}{g_{p, b, c}^{\prime}(z)}-\frac{2 z g_{p, b, c}^{\prime}(z)}{g_{p, b, c}(z)}\right\}<\frac{c+d}{(1+c)(1-d)},
$$

then $g_{p, b, c}(z)$ is univalent in $\mathbb{U}$.

Proof. Define a function $q(z)$ as follows:

$$
\frac{z^{2} g_{p, b, c}^{\prime}(z)}{\left(g_{p, b, c}(z)\right)^{2}}=\frac{1+a q(z)}{1-b q(z)} \quad(z \in \mathbb{U})
$$

We see that $q(z)$ is analytic in $\mathbb{U}$ and $q(0)=0$. Differentiation of (54) gives

$$
\begin{aligned}
& \frac{\left[z g_{p, b, c}(z)\right]^{\prime \prime}}{g_{p, b, c}^{\prime}(z)}-\frac{2 z g_{p, b, c}^{\prime}(z)}{g_{p, b, c}(z)} \\
& =\frac{(c+d) z q^{\prime}(z)}{(1+c q(z))(1-d q(z))}=E_{5}(z), \text { say. }
\end{aligned}
$$

Now suppose there exists $z_{0} \in \mathbb{U}$ such that

$$
\max _{|z|<\left|z_{0}\right|}|q(z)|=\left|q\left(z_{0}\right)\right|=1 .
$$

Then from Lemma 1, we have

$$
z_{0} q^{\prime}\left(z_{0}\right)=k q\left(z_{0}\right) .
$$


Now letting $q\left(z_{0}\right)=e^{i \theta},(\theta \in[0,2 \pi])$ in (55), we have

$$
\begin{aligned}
& \operatorname{Re}\left(E_{5}\left(z_{0}\right)\right)=k(a+b) \\
& \cdot \operatorname{Re}\left(\frac{q\left(z_{0}\right)}{\left(1+c q\left(z_{0}\right)\right)\left(1-d q\left(z_{0}\right)\right)}\right)=k \\
& \cdot \operatorname{Re}\left(\frac{1}{\left(1-d q\left(z_{0}\right)\right)}-\frac{1}{\left(1+c q\left(z_{0}\right)\right)}\right)=k \\
& \cdot \operatorname{Re}\left(\frac{1-d e^{i \theta}}{1+d^{2}-2 d \cos \theta}-\frac{1+c e^{i \theta}}{1+c^{2}+2 c \cos \theta}\right) \\
& =k\left(\frac{1}{2+\left(d^{2}-1\right) /(1-d \cos \theta)}\right. \\
& \left.-\frac{1}{2+\left(c^{2}-1\right) /(1+c \cos \theta)}\right)
\end{aligned}
$$

where $\theta \neq \cos ^{-1}(-1 / c)$ and $\theta \neq \cos ^{-1}(1 / d)$. Since $k \geq 1$ we have

$$
\operatorname{Re}\left(E\left(z_{0}\right)\right)>\frac{c+d}{(1+c)(1-d)} .
$$

This contradicts the hypothesis and therefore $|q(z)|<1$ for all $z \in \mathbb{U}$. Thus,

$$
\left|\frac{z^{2} g_{p, b, c}^{\prime}(z)}{\left(g_{p, b, c}(z)\right)^{2}}-1\right|=\left|\frac{(c+d) q(z)}{1-d q(z)}\right|<\frac{c+d}{1-d} \leq 1
$$

$$
(z \in \mathbb{U})
$$

In view of Lemma 2 it implies that $g_{p, b, c}$ is in $\mathcal{U}$ and hence univalent.

Theorem 10. Let $M \geq 1, v$ be a real number such that $v \geq$ $2 M+1$ and let $\gamma$ be a complex number which satisfies the inequality

$$
|\gamma| \leq 1-\frac{1}{\nu}(2 M+1)
$$

If $g_{p, b, c}$ is univalent in $\mathbb{U}$ and $\left|g_{p, b, c}(z)\right| \leq M$ for all $z \in \mathbb{U}$, then the function

$$
E(z)=\left\{v \int_{0}^{z} t^{\nu-1} \frac{g_{p, b, c}(t)}{t} d t\right\}^{1 / v}
$$

is univalent in $\mathbb{U}$, where the values of the complex powers are taken with their principal values.

Proof. Define a function

$$
p(z)=\int_{0}^{z} \frac{g_{p, b, c}(t)}{t} d t .
$$

Then we have $p(0)=p^{\prime}(0)-1=0$.
Also

$$
\begin{aligned}
p^{\prime}(z) & =\frac{g_{p, b, c}(z)}{z}, \\
\frac{z p^{\prime \prime}(z)}{p^{\prime}(z)} & =\frac{z g_{p, b, c}^{\prime}(z)}{g_{p, b, c}(z)}-1 .
\end{aligned}
$$

From (65), we have

$$
\begin{aligned}
\left|\frac{z p^{\prime \prime}(z)}{p^{\prime}(z)}\right| & \leq\left|\frac{z g_{p, b, c}^{\prime}(z)}{g_{p, b, c}(z)}\right|+1 \\
& =\left|\frac{z^{2} g_{p, b, c}^{\prime}(z)}{\left(g_{p, b, c}(z)\right)^{2}}\right|\left|\frac{g_{p, b, c}(z)}{z}\right|+1 .
\end{aligned}
$$

From the hypothesis, we have $\left|g_{p, b, c}(z)\right| \leq M(z \in \mathbb{U})$; then, by the Schwarz Lemma (cf [17], we obtain that

$$
\left|g_{p, b, c}(z)\right| \leq M|z| \quad(z \in \mathbb{U}) .
$$

Now, since $g_{p, b, c}(z)$ is univalent in $\mathbb{U}$

$$
\begin{aligned}
\left|\frac{z p^{\prime \prime}(z)}{p^{\prime}(z)}\right| & \leq\left|\frac{z^{2} g_{p, b, c}^{\prime}(z)}{\left(g_{p, b, c}(z)\right)^{2}}\right| M+1 \\
& \leq\left|\frac{z^{2} g_{p, b, c}^{\prime}(z)}{\left(g_{p, b, c}(z)\right)^{2}}-1\right| M+M+1 \leq 2 M+1 .
\end{aligned}
$$

Using (68), we have

$$
\left.|\gamma| z\right|^{2 v}+\left(1-|z|^{2 v}\right) \frac{z p^{\prime \prime}(z)}{v p^{\prime}(z)}|\leq| \gamma\left|+\frac{1}{v}\right| \frac{z p^{\prime \prime}(z)}{v p^{\prime}(z)} \mid
$$

$$
\leq|\gamma|+\frac{1}{\beta}(2 M+1) .
$$

So, from (61) we have

$$
\left.|\gamma| z\right|^{2 v}+\left(1-|z|^{2 v}\right) \frac{z p^{\prime \prime}(z)}{v p^{\prime}(z)} \mid \leq 1 .
$$

Applying Lemma 3, we obtain the function $E(z)$ defined by (62) which is univalent in $\mathbb{U}$.

\section{Competing Interests}

The authors declare that there is no conflict of interests regarding the publication of this paper.

\section{Acknowledgments}

The work of the first author was supported by the Department of Science and Technology, India, with reference to the Sanction Order no. SR/DST-WOS A/MS-10/2013(G). The work of the second author was supported by the grant given under UGC Minor Research Project F. no. 5599/15 (MRP-SEM/UGC SERO). The work of the third author was supported by the grant given under UGC Minor Research Project, 201415/MRP-5591/15(SERO/UGC). 


\section{References}

[1] P. J. Eenigenburg, "A class of starlike mappings of the unit disk," Compositio Mathematica, vol. 24, pp. 235-238, 1972.

[2] H. Silverman, "Subclasses of starlike functions," Revue Roumaine de Mathématique Pures et Appliquées, vol. 23, no. 7, pp. 1093-1099, 1978.

[3] D. J. Wright, "On class of starlike functions," Compositio Mathematica, vol. 21, pp. 122-124, 1969.

[4] L. A. Aksentev, "Sufficient conditions for univalence of regular functions," Izvestiya Vysshikh Uchebnykh Zavedenii Matematika, no. 4, pp. 3-7, 1958 (Russian).

[5] M. Obradović, S. Ponnusamy, and K. J. Wirths, "Geometric Studies on the Class $U(\lambda)$, , Bulletin of the Malaysian Mathematical Sciences Society Series 2, vol. 39, no. 3, pp. 1259-1284, 2016.

[6] M. Obradovic, "A class of univalent functions," Hokkaido Mathematical Journal, vol. 27, no. 2, pp. 329-335, 1998.

[7] M. Obradovic and S. Ponnusamy, "New criteria and distortion theorems for univalent functions," Complex Variables, Theory and Application, vol. 44, no. 3, pp. 173-191, 2001.

[8] M. Obradovic and S. Ponnusamy, "Univalence and starlikeness of certain transforms defined by convolution of analytic functions," Journal of Mathematical Analysis and Applications, vol. 336, no. 2, pp. 758-767, 2007.

[9] N. Yagmur and H. Orhan, "Starlikeness and convexity of generalized Struve functions," Abstract and Applied Analysis, vol. 2013, Article ID 954513, 6 pages, 2013.

[10] H. Orhan and N. Yagmur, "Geometric properties of generalized Struve functions," in Proceedings of the International Congress in Honour of Professor Hari M. Srivastava, Bursa, Turkey, August 2012.

[11] S. P. Goyal, R. Kumar, and T. Bubloaca, "Majorization problems and integral transforms for a class of univalent functions with missing coefficients," Boletim da Sociedade Paranaense de Matemática, vol. 33, no. 2, pp. 217-230, 2015.

[12] M. Obradovic and S. Ponnusamy, "Univalency and convolution results associated with confluent hypergeometric functions," Houston Journal of Mathematics, vol. 35, no. 4, pp. 1313-1328, 2009.

[13] P. Hästö, S. Ponnusamy, and M. Vuorinen, "Starlikeness of the Gaussian hypergeometric functions," Complex Variables and Elliptic Equations, vol. 55, no. 1-3, pp. 173-184, 2010.

[14] S. S. Miller and P. T. Mocanu, "Second order differential inequalities in the complex plane," Journal of Mathematical Analysis and Applications, vol. 65, no. 2, pp. 289-305, 1978.

[15] M. Nunokawa, "On some angular estimates of analytic functions," Mathematica Japonica, vol. 41, no. 2, pp. 447-452, 1995.

[16] S. S. Miller and P. T. Mocanu, "Differential subordinations and inequalities in the complex plane," Journal of Differential Equations, vol. 67, no. 2, pp. 199-211, 1987.

[17] Z. Nehari, Conformal Mapping, McGraw Hill Book Comp, New York, NY, USA, 1952, (Dover, 1975). 


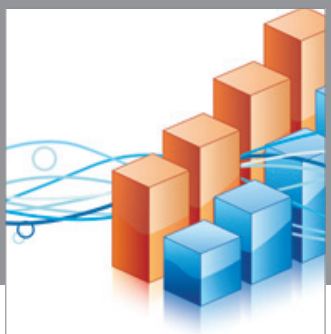

Advances in

Operations Research

vatem alat4

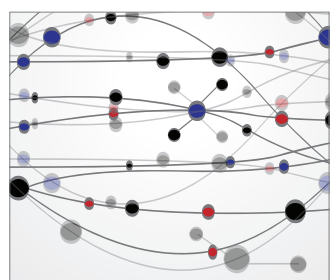

\section{The Scientific} World Journal
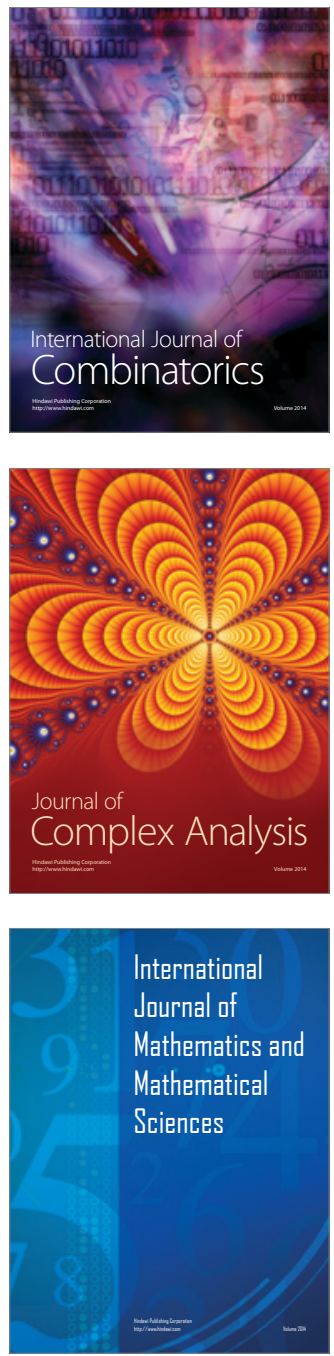
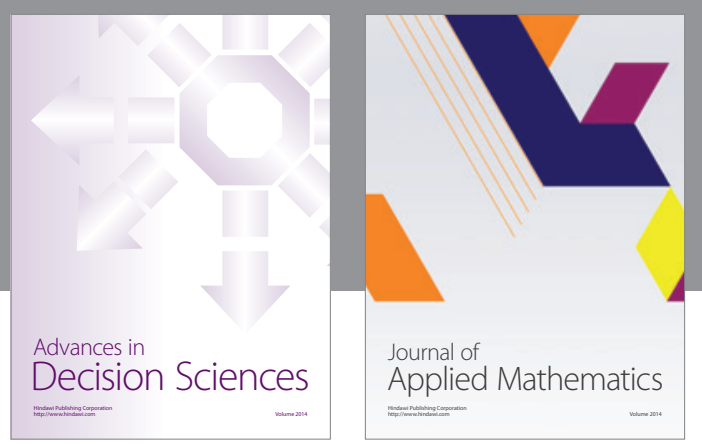

Algebra

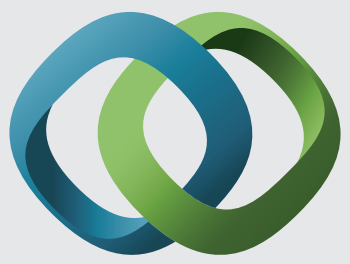

\section{Hindawi}

Submit your manuscripts at

http://www.hindawi.com
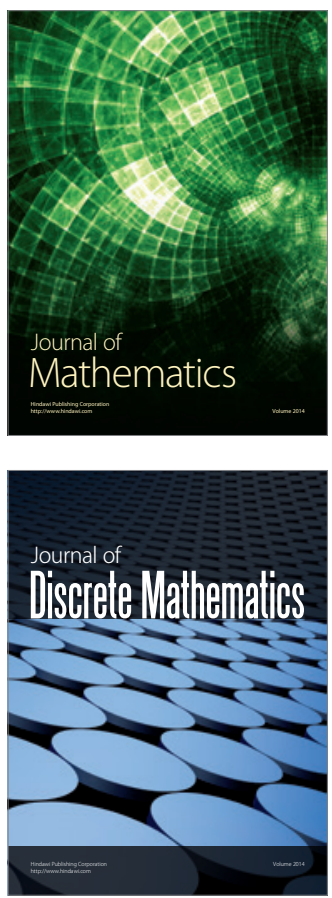

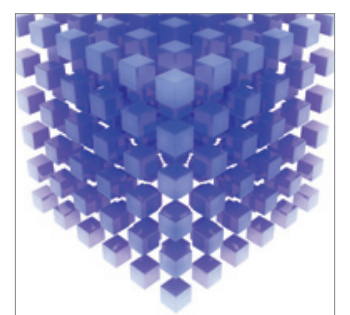

Mathematical Problems in Engineering
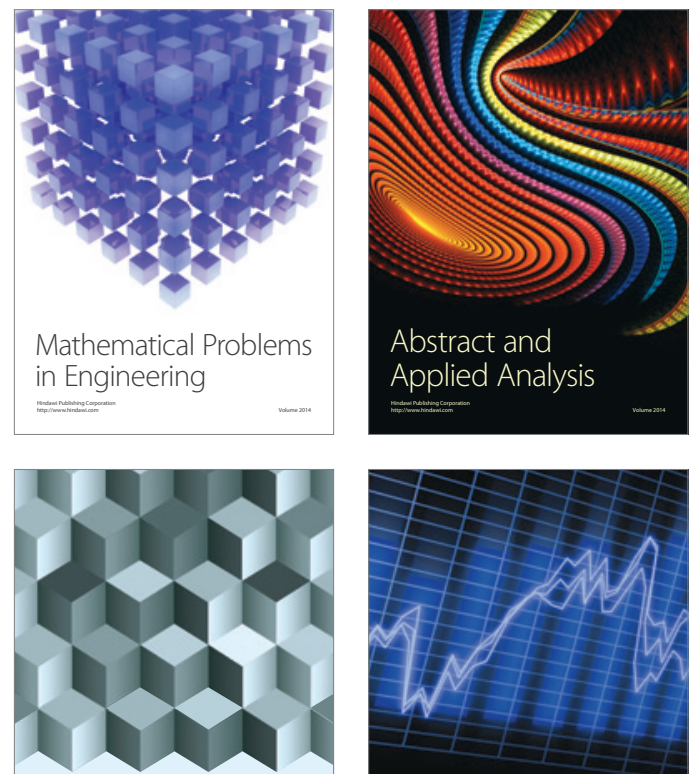

Journal of

Function Spaces

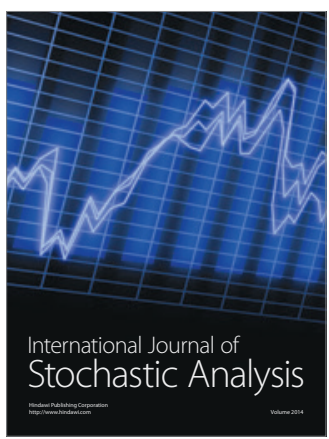

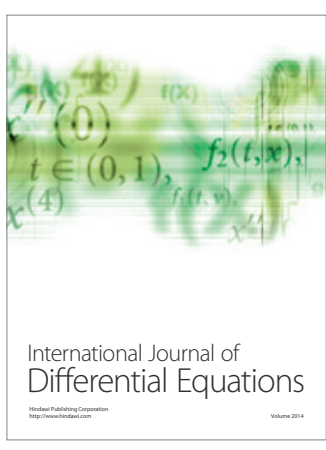
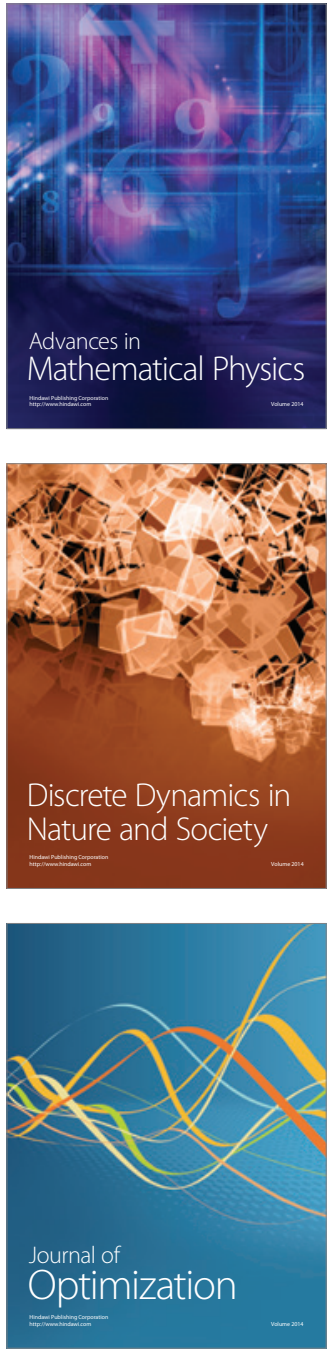